\title{
ADAPTIVE BEAMFORMING FOR SLOW FADING RAYLEIGH SIGNALS
}

\author{
R. DeLap \\ Dept of EENG \\ Air Force Institute of Technology \\ Wright-Patterson AFB, OH 45433
}

\section{ABSTRACT}

In this paper we develop an adaptive beamsummer for direction of arrival (DOA) estimation of slow fading Raleigh signals, using a new design approach, termed "Adaptive Detection/Estimation for sPecific Tasks" (ADEPT). For DOA estimation, the ADEPT method yields a weight adaptation criterion which is optimized for those weights that minimize the Cramer-Rao (CR) lower bound on achievable mean-square-crror of any unbiased DOA estimator constructed on the beamsummer outputs. Simulation results are provided which show that the ADEPT DOA beamsummer yields DOA estimates whose meansquared error (MSE) approaches that of the more complex maximum likelihood implementation.

\section{PROBLEM STATEMENT}

Figure 1 shows an $m$-element linear beamsumming array on which impinge $p$ signals arriving from directions $\theta_{1}, \ldots, \theta_{p}$. At the $k$-th time instant a snapshot $\underline{Y}^{k}=$ $\left[Y_{1}^{k}, \ldots, Y_{m}^{k}\right]^{T}$ of the $m$-sensors is acquired, $k=1, \ldots, n$. We assume the following model for slow Rayleigh fading [1] in the received signals: for each signal the amplitudes are perfectly coherent over the array for any particular snapshot but vary randomly for different snapshots. In addition we assume that the ambient noise $\underline{N}^{k}=\left[N_{1}^{k}, \ldots, N_{m}^{k}\right]^{T}$ is spatially incoherent and temporally uncorrelated. Under the above assumptions we have the model for the beamsummer output $x^{k}$ :

$$
x^{k}=\underline{\mathrm{W}}^{H} \mathrm{~V}(\theta) \underline{S}^{k}+\underline{\mathrm{W}}^{H} \underline{N}^{k}
$$

where $S=\left[s_{1}, \ldots, s_{p}\right]^{T}$ is a vector of complex signal amplitudes, and $V$ is an $m \times p$ matrix of steering vectors. With this model the mean and covariance of the $k^{\text {th }}$ snapshot are:

$$
\begin{aligned}
\mu_{x}=E\left[x^{k}\right] & =\underline{\mathrm{W}}^{H} \mathrm{~V} \underline{\mu}_{S} \\
\sigma_{x}=\operatorname{cov}(x) & =\underline{\mathrm{W}}^{H} \mathrm{~V} \Sigma_{S} \mathrm{~V}^{H} \underline{\mathrm{W}}+\underline{\mathrm{W}} \Sigma_{N} \underline{\mathrm{W}}
\end{aligned}
$$

where $\Sigma_{N}=\sigma_{N}^{2} \mathrm{I}$ is the covariance of the array noise.

\section{OPTIMAL BEAMSUMMING FOR DOA-ESTIMATION}

Here we review the ADEPT-DOA method of optimal weight design for DOA estimation using Fisher information and the Cramer-Rao (CR) bound [2].

\author{
A. Hero \\ Dept of EECS \\ The University of Michigan \\ Ann Arbor, MI 48109
}

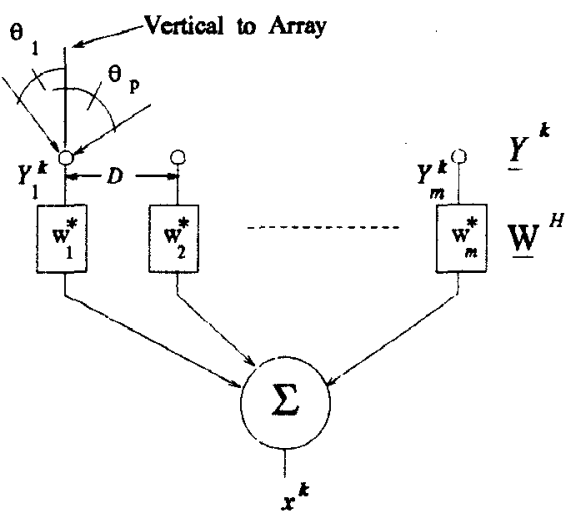

Figure 1: Beamsummer Model.

Let $\phi$ be an unknown parameter of interest. For fixed beamsummer weights $W$ let the $N$ snapshots of the beamsummer outputs $\underline{x}=\left[x^{1}, \ldots, x^{N}\right]^{T}$ have joint probability density function $f_{\underline{x}}(\underline{x} ; \phi)$ parameterized by the unknown scalar parameter $\phi$. Define the Fisher information:

$$
F_{\phi}=E_{\phi}\left[\frac{\partial}{\partial \phi} \ln f_{\underline{x}}(\underline{x} ; \phi) \frac{\partial}{\partial \phi} \ln f_{\underline{x}}(\underline{x} ; \phi)\right] \text {. }
$$

Note that the Fisher information is a function of both $\phi$ and $\underline{W}$. Under appropriate regularity conditions, any unbiased estimator $\hat{\phi}=\hat{\phi}(\underline{x})$ of $\phi$ has variance which satisfies the lower bound $\operatorname{var}[\hat{\phi}] \geq \frac{1}{F_{\phi}}$. The methodology of optimal design of experiments attempts to maximize the Fisher information $F_{\phi}=F_{\phi}(\underline{\mathrm{W}})$ over $\underline{\mathrm{W}}$ in order to provide data $\underline{x}$ which allows the most accurate parameter estimation performance. For a single signal, the Fisher information for the signal direction of arrival $\phi=\theta$ can be reduced to the form [2]:

$$
\begin{aligned}
F_{\theta}= & {\left[\left(\frac{\underline{\widetilde{W}}^{H}\left[\underline{V}_{p} \underline{V}^{H}+\underline{V} \underline{V}_{p}^{H}\right] \underline{\tilde{W}}}{\|\underline{\underline{W}}\|^{2}}\right)^{2} \cdot \frac{1}{m^{2}} \delta_{o}^{2}\right.} \\
& \left.+2\left(\frac{\underline{\tilde{W}}^{H}\left[\underline{V}_{p} \underline{V}_{p}^{H}\right] \underline{\tilde{W}}}{\|\underline{\widetilde{W}}\|^{2}}\right) \cdot \frac{1}{m} \gamma_{o}^{2}\right] \cdot n u^{2} \cos ^{2} \theta
\end{aligned}
$$

where $u=D / \lambda, D$ is the interelement spacing, $\lambda$ is the signal wavelength, and

$$
\underline{V}=\left[e^{j^{\frac{2 l-m-1}{2}} \mathrm{usin} \theta}\right]_{l=1}^{m},
$$




$$
\begin{aligned}
\mathrm{B} & =\operatorname{diag}\left(j \frac{2 l-m-1}{2}\right)_{l=1}^{m}, \\
\underline{V_{p}} & =\mathrm{B} \underline{V}, \\
\underline{\tilde{\mathrm{W}}} & =\left[\underline{V} V^{H} \sigma_{s}^{2}+1_{m} \sigma_{N}^{2}\right]^{\frac{1}{2}} \underline{\mathrm{W}},
\end{aligned}
$$

$I_{m}$ is the $m \times m$ identity matrix, and

$$
\begin{aligned}
\gamma_{o}^{2} & =\frac{m\left|\mu_{s}\right|^{2}}{\sigma_{N}^{2}}, \\
\delta_{o}^{2} & =\alpha_{o}^{2} \frac{\alpha_{o}^{2}}{1+\alpha_{o}^{2}}, \\
\alpha_{o}^{2} & =\frac{m \sigma_{s}^{2}}{\sigma_{N}^{2}} .
\end{aligned}
$$

Note that $\delta_{0}^{2}$ is monotone in $\alpha_{o}^{2}$, which is the maximum signal variance to noise ratio of the output of the beamsummer. The maximum $\alpha_{0}^{2}$ is achieved by the adapted Frost beamsummer [3]. Also, $\gamma_{0}^{2}$ is the maximum squared signal mean to noise ratio of the output of the beamsummer. The maximum $\gamma_{0}^{2}$ is achieved by the adapted Applebaum beamsummer [4]. The ADEPT-DOA method for beamsumming seeks to choose the weight vector $\underline{W}$ which maximizes the Fisher information. By maximizing $F_{\theta}(\underline{W})$ with respect to $\underline{W}$ we achieve an optimal compromise between $\alpha_{o}^{2}$ and $\gamma_{o}^{2}$ by minimizing the CR bound on unbiased DOA estimator variance at the beamsummer output.

Define the normalized vectors $\underline{\hat{V}}=\underline{V} /\|\underline{V}\|$ and $\underline{\tilde{V}}_{p}=$ $\underline{V}_{p} /\left\|\underline{V}_{p}\right\|$. In [2] we show that the optimal weight vector that maximizes $F_{\theta}$ is given up to a complex scale factor by:

$$
\underline{\mathrm{W}}_{o p t}= \begin{cases}\widetilde{V}_{p}+a \underline{\tilde{V}}, & \delta_{o}^{2}>\gamma_{o}^{2} / 2 \\ \underline{\widetilde{V}}_{p}, & \delta_{o}^{2} \leq \gamma_{o}^{2} / 2\end{cases}
$$

where

$$
a= \pm \frac{1}{1+\alpha_{o}} \sqrt{\frac{2 \delta_{o}^{2}-\gamma_{o}^{2}}{2 \delta_{o}^{2}+\gamma_{o}^{2}}} .
$$

The following interpretations follow directly from these results:

- The optimal weight vector consists of the sum of the two unity normalized orthogonal components $\widetilde{V}$ and $\tilde{V}_{p}$. It is easily seen that $\underline{W}=\underline{V}$ gives a beamsummer which maximizes mean array gain in the direction of the signal. On the other hand, $\underline{W}=\underline{V}_{p}$ gives a beamsummer which is a "signal nuller" - it minimizes mean array gain in the direction of the signal.

- For $\delta_{o}^{2} \leq \gamma_{o}^{2} / 2$, up to an arbitrary scale factor, $\underline{W}_{o p t}=\underline{V}_{p}$, i.e. the optimal weight vector places a null exactly at the signal angle $\theta$. This suggests that for this case, the best $\theta$-estimator performance is achieved by trying to null out the signal, e.g. by minimizing beamsummer response. On the other hand, when the signal energy is concentrated in the signal variance $\left(\alpha_{o}^{2} \gg \gamma_{o}^{2}\right)$ the Fisher information indicates that the optimal weights must pass some signal energy - adding or subtracting $a \underline{\underline{V}}$ from $\underline{\tilde{V}}_{p}$ accomplishes this.
$\underline{W}_{\text {opt }}$ given in (12) is unimplementable since it requires knowledge of $\theta$ and the SNR-dependent constant $a$. We develop a direct implementation method in [2] which shows that maximization of $F_{\theta}(\underline{\mathrm{W}})$ is equivalent to maximization of the function $F_{D O A}(\underline{\mathrm{W}})$ :

$$
F_{D O A}(\underline{\mathrm{W}})=\frac{\widetilde{\widetilde{W}}^{H}\left[G_{1} K_{1}(\underline{\widetilde{W}})+G_{2} K_{2}(\underline{\tilde{W}})\right] \underline{\tilde{W}}}{\underline{\tilde{W}}^{H} \underline{\tilde{W}}}
$$

where

$$
\begin{aligned}
& \underline{\tilde{\mathrm{W}}}=\operatorname{cov}^{\frac{1}{2}}[\underline{Y}] \underline{\mathrm{W}} \\
& G_{1}=\operatorname{cov}^{\frac{1}{2}}[\underline{Y}] \mathrm{B}_{1}^{H} \operatorname{cov}^{-\frac{1}{2}}[Y]+\operatorname{cov}^{-\frac{1}{2}}[Y] \mathrm{B}_{1} \operatorname{cov}^{\frac{1}{2}}[\underline{Y}] \\
& G_{2}=4 m \cdot \operatorname{cov}^{-\frac{1}{2}}[Y] B_{1} E[Y] E\left[Y^{H}\right] \mathrm{B}_{1}^{H} \operatorname{cov}^{-\frac{1}{2}}[Y] \\
& K_{1}(\underline{\widetilde{W}})=\frac{\widetilde{\mathrm{W}}^{H} G_{1} \tilde{\tilde{W}}}{\widetilde{\mathrm{W}}^{H} \underline{\underline{\mathrm{W}}}}, \\
& K_{2}(\underline{\tilde{W}})=1 \text {, }
\end{aligned}
$$

and $\mathrm{B}_{1}$ and $\mathrm{B}_{2}$ are the following $m \times m$ complex matrices:

$$
\begin{aligned}
& \mathrm{B}_{1 .}=\operatorname{diag}_{l=1, \ldots, m}\left(j \frac{(2 l-m-1) / 2}{\sqrt{m\left(m^{2}-1\right) / 6}}\right) \\
& \mathrm{B}_{2}=\operatorname{diag}\left(\frac{1}{\sqrt{2 m}}\right)
\end{aligned}
$$

Note that $F_{D O A}(\underline{\mathrm{W}})$ can be used for weight adaptation by substituting sample means and covariances for $E[Y]$ and $\operatorname{cov}[Y]$ and maximizing over $\widetilde{W}$. An iterative algorithm for this maximization is given below:

Eigenmaximization Algorithm

\{nitialize: $\widetilde{\widetilde{W}}(0)=$ init. weights, $\nu=$ tol, $i=1$. while $\|\underline{\tilde{W}}(i+1)-\underline{\widetilde{W}}(i)\|>\nu$

\{

$$
\underline{\widetilde{W}}(i+1)=\max _{\text {e-vector }}\left[G_{1} K_{1}(\underline{\tilde{W}}(i))+G_{2} K_{2}(\underline{\tilde{W}}(i))\right]
$$$$
i=i+1
$$

\}

\}

$\underline{W}=\operatorname{cov}^{-\frac{1}{2}}[\underline{Y}] \underline{\widetilde{W}}(i+1)$

This algorithrn requires finding the square root factors $\operatorname{cov}^{\frac{1}{2}}[Y]$ and $\operatorname{cov}^{-\frac{1}{2}}[Y]$, e.g. by Cholesky decomposition, and finding the eigenvector associated with the largest eigenvalue of the matrix $G_{1} K_{1}(\underline{\widetilde{W}})+G_{2} K_{2}(\underline{\widetilde{W}})$. A steepest descent algorithm can also be derived which reduces the computational load but only guarantees convergence to a local maximum of $F_{D O . A}(\underline{W})$ [5]. A block diagram of the DOA-optimal beamsummer is shown in Figure 2.

\section{NULL PICKING FOR DOA ESTIMATION}

We use a very simple gated null picking method to estimate the DOAs from the adaptive weights given by the above eigenmaximization algorithm. We begin by computing the 


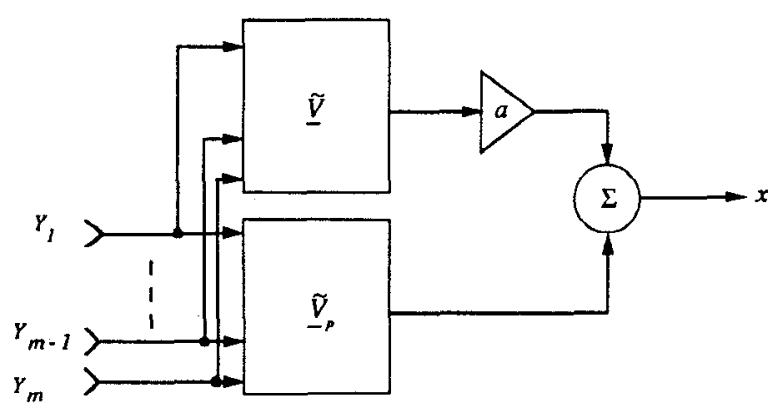

Figure 2: DOA-optimal Beamsummer Block Diagram.

discrete fourier transform (DFT) of the adapted DOAoptimal weight vector $\underline{W}$, which yields an antenna gain pattern for the adapted weights [2,5]. Nulls in this beampattern represent possible signal DOAs. To determine which nulls represent signal DOAs, we begin by implementing a signal detection-optimal (D-optmal) beamsumming method which is developed in $[2,5]$, called ADEPT$D$. The D-optimal beamsummer maximizes the function $F_{D}(\underline{W})$ over the weight vector $\underline{W}$ :

$$
F_{D}(\underline{\mathrm{W}})=\frac{\underline{\mathrm{W}}^{H}\left[G_{1} K_{1}(\underline{\mathrm{W}})+G_{2} K_{2}(\underline{\mathrm{W}})\right] \underline{\mathrm{W}}}{\underline{\mathrm{W}}^{H} \underline{\mathrm{W}}}
$$

where

$$
\begin{aligned}
G_{1} & =E[\underline{Y}] E\left[\underline{Y}^{H}\right] \\
G_{2} & =\operatorname{cov}[\underline{Y}]-\sigma_{N}^{2}, \\
K_{1}(\underline{\mathrm{W}}) & =1+2\left(1+\alpha^{2}\right)+\gamma^{2}\left[\frac{\left(1+\alpha^{2}\right)^{2}}{\alpha^{4}+\gamma^{2}}\right] \\
K_{2}(\underline{\mathrm{W}}) & =\alpha^{2} \\
\gamma^{2} & =\frac{\underline{\mathrm{W}}^{H}\left[G_{1}\right] \underline{\mathrm{W}}}{\underline{\mathrm{W}}^{H} \underline{\mathrm{W}}} \cdot \frac{1}{\sigma_{N}^{2}} \\
\alpha^{2} & =\frac{\underline{\mathrm{W}}^{H}\left[G_{2}\right] \underline{\mathrm{W}}}{\underline{\mathrm{W}^{H}}} \cdot \frac{1}{\sigma_{N}^{2}} .
\end{aligned}
$$

The D-optimal beamsummer is implemented using the previously described eigenmaximization algorithm. The resulting D-optimal weight vector produces peaks in the Doptimal antenna gain pattern near the DOAs of the incoming signals. We search the DOA-optimal beampattern for nulls which lie within 1 beamwidth "gates" of these peaks. The deepest null within each of these gates is used as a DOA estimate. This process is illustrated in Figure 3.

\section{SIMULATION RESULTS}

An i.i.d. Gaussian sequence of zero mean noise variates was generated with variance $\sigma_{n}^{2}=1$, as was an i.i.d. Gaussian sequence of signal variates with prescribed mean $\mu_{\text {s }}$ and variance $\sigma_{s}^{2}$. From the signal and noise sequences, 1000 realizations of groups of 100 snapshots of the output of a six element uniform linear array were synthesized. For Simulation I, we set $\gamma_{o}^{2}=60$ (eq. 9), $\alpha_{o}^{2}=6$ (eq.

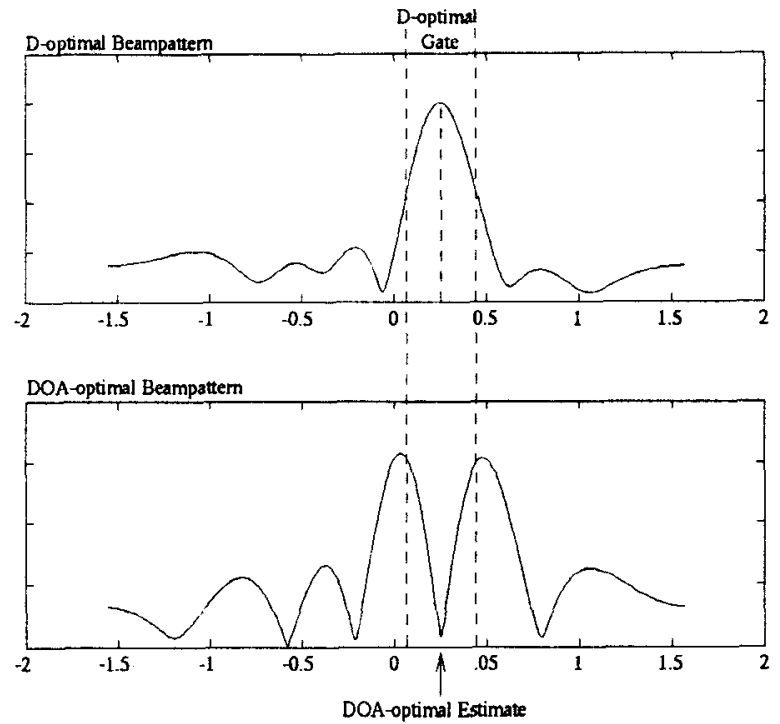

Figure 3: DOA Estimation via Gated Null Picking for a Signal Arriving at $\theta=.25$ Radians.

\begin{tabular}{||c|c|c|c||}
\hline Simulation & $\gamma_{o}^{2}$ & $\alpha_{o}^{2}$ & $\theta$ \\
\hline \hline I & 60 & 6 & .22 radians \\
\hline II & 6 & 60 & .22 radians \\
\hline
\end{tabular}

Table 1: Simulation Signal Parameters

11). For simulation II we set $\gamma_{o}^{2}=6, \alpha_{o}^{2}=60$. A summary of the signal parameters used for the simulations is given in Table 1 . Figure 4 shows a typical realization of adaptive beamsummer beampatterns for Simulation $I$ and varying numbers of snapshots for the Applebaum and DOA-optimal beamsummers. Also shown are the ensemble average beampatterns. The vertical dashed line represents the true DOA. Note the sharpness of the null in the DOA-optimal beampatterns near the true signal DOA. Figures 5-6 show the MSE of the DOA-estimates for the Applebaum peak picking and DOA-optimal gated null picking estimators. For comparison purposes, a clairvoyant maximum-likelihood (ML) DOA estimator was implemented, operating on the raw array data. This estimator was implemented by maximizing the array likelihood function $f\left(\underline{Y} \mid \underline{\mu}_{S}, \Sigma_{S}, \sigma_{N}^{2}, \theta\right)$ over $\theta$ assuming that the true values for $\underline{\mu}_{S}, \Sigma_{S}$, and $\sigma_{N}^{2}$ are known without error. The MSE of DOA estimates obtained using the ML estimator is included in Figures 5-6. Finally, we plot the minimum beamsummer CR-bound $1 / \max _{\underline{W}} F_{\theta}(\underline{W})$ on MSE for any unbiased estimator. Note that the clairvoyant ML MSE falls below the beamsummer CR bound, as expected. However, since the ML MSE is only slightly below the CR bound, only a small loss in estimator performance is incurred by restricting ourselves to a beamsummer based estimator implementation. Also, note that the MSE in the DOA-optimal estimates approaches that of the clairvoyant $M L$ estimator and the beamsummer $C R$ bound as 

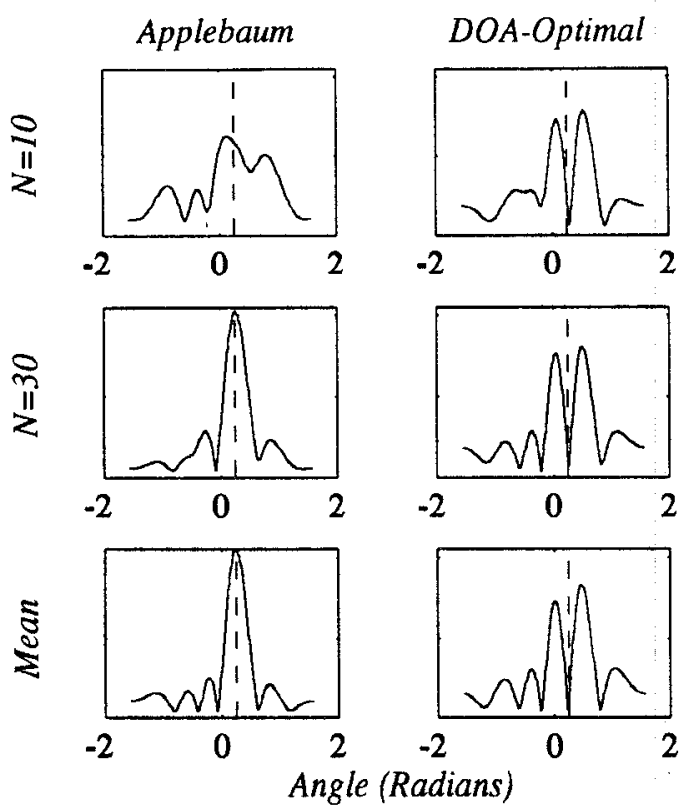

Figure 4: Typical Beampatterns for a Single Realization.

the number of snapshots $n$ becomes large. The improved estimation performance of the DOA-optimal beamsummer over the Applebaum beamsummer can be attributed to the DOA-optimal beamsummer's being based upon minimizing the CR bound.

\section{CONCLUSIONS}

We have introduced a new method of beamformer design, (ADEPT), which adapts the beamformer weights to provide the minimum $C R$ bound for DOA estimation based on the beamsummer outputs. Our simulations show that when implemented with a very simple post-beamsumming DOA estimation technique, the DOA-optimal beamsummer approaches the CR bound on MSE for any unbiased DOA estimation technique implemented at the beamsummer output. In $[2,5]$, we introduce other optimal beamsummers designed using the ADEPT method for the purposes of signal detection and estimation of constant modulus signal parameters. More extensive simulation results can be found in [5] for multiple signals and wideband beamsummer arrays.

\section{REFERENCES}

[1 ] J. I. Marcum and P. Swerling, "Studies of target detection by pulsed radar," IRE Trans. on Inform. Theory, vol. IT-6, pp. 59-308, April 1960.

[2] A. O. Hero and R. A. DeLap, "Task specific criteria for adaptive beamforming with slow fading signals," in Recent Advances in Array Signal Processing, S. Haykin, ed., Prentice Hall, (to be published 1994).

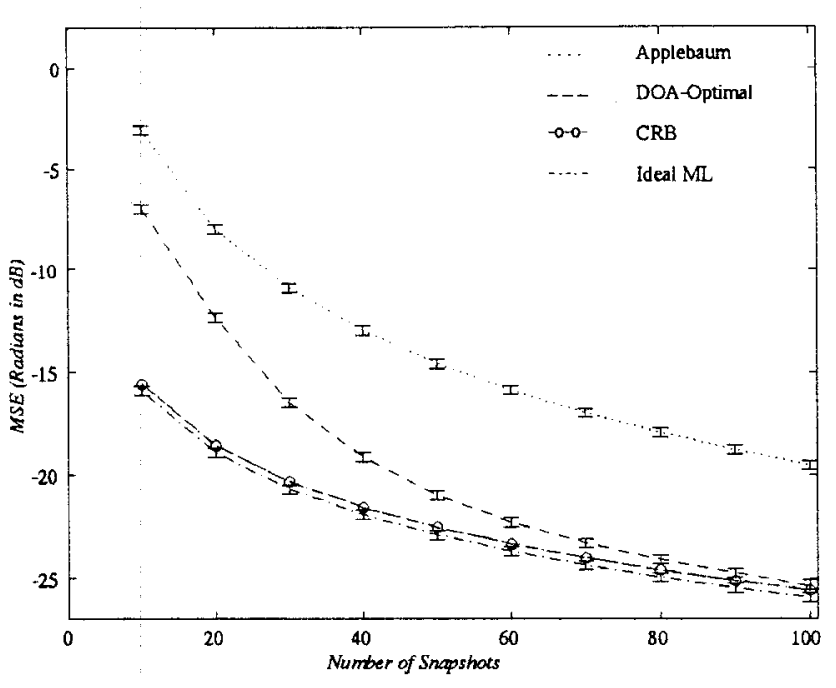

Figure 5: MSE In DOA Estimates: Simulation I.

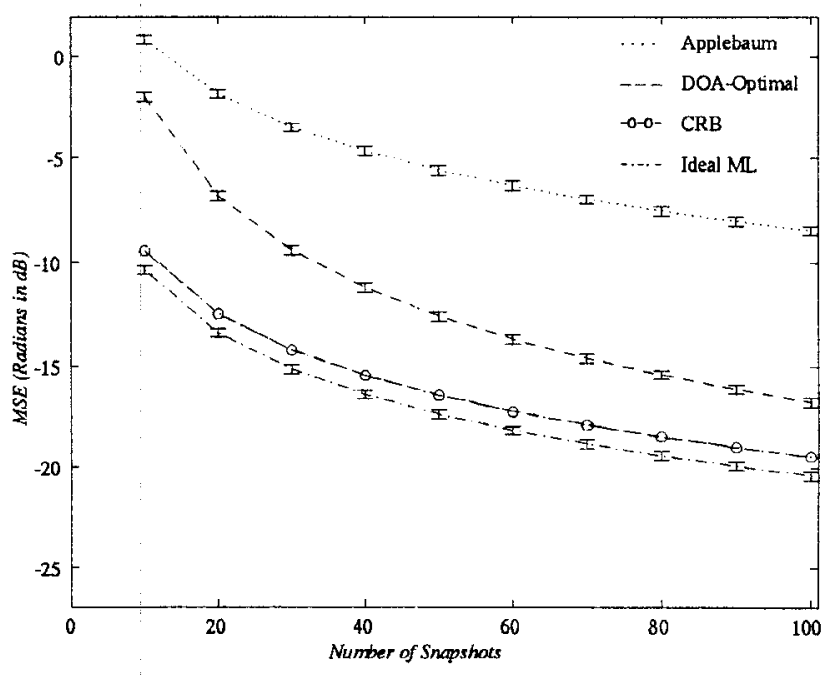

Figure 6: MSE In DOA Estimates: Simulation II.

[3 ] O. L. Frost III, "An algorithm for linearly constrained adaptive array processing," Proc. IEEE, vol 60, August 1972, pp926-35.

[4 ] S. P. Applebaum, "Adaptive arrays," IEEE Trans. Antennas and Propagation, vol. AP-24, pp. 585-598, Sept. 1976.

[5] R. A. DeLap, ADEPT: Task Specific Adaptive Beamforming, PhD thesis, University of Michigan, Ann Arbor, MI, 1994. 